\title{
Toward an effective use of $\beta$-catenin immunohistochemistry in the evaluation of challenging melanocytic lesions
}

\author{
Emily M. Erstine ${ }^{1}$ (D) - Alexander J. Lazar ${ }^{1,2,3,4}$
}

Received: 21 February 2019 / Accepted: 26 February 2019 / Published online: 13 March 2019

(C) Springer-Verlag GmbH Germany, part of Springer Nature 2019

In this issue, Fouchardiere and colleagues [1] proffer that nuclear $\beta$-catenin expression as detected by immunohistochemistry (IHC) can be a helpful screening tool to distinguish an ambiguous dermal melanocytic proliferation as a deep penetrating nevus (DPN) from similar entities on its differential diagnosis, including "blue" melanocytic tumors, spitzoid tumors, nevoid and superficial spreading melanomas (SSM), and pigmented epithelioid melanocytomas (PEM). Firstly, they retrospectively evaluated nuclear $\beta$-catenin expression in 178 diagnostically challenging and unambiguous lesions and found that $98 / 100$ of the DPN were positive, along with 2/16 melanomas (one SSM and one nevoid melanoma with a plexiform clone), while all spitzoid, blue, and $\operatorname{PEM~}(n=30,26$ and 6 , respectively) lesions lacked nuclear accumulation of $\beta$ catenin. Because nuclear accumulation of $\beta$-catenin can drive cyclin D1 expression in other cellular contexts, they also evaluated nuclear and cytoplasmic cyclin D1 expression, which was not significantly different among the diagnostic groups, suggesting that cyclin D1 expression is not entirely dependent upon nuclear $\beta$-catenin expression. Secondly, they evaluated nuclear $\beta$-catenin IHC expression in a set of 13 diagnostically-ambiguous melanocytic tumors with DPN in the differential diagnosis. Three of four cases with canonical DPN mutation profiles were the only $\beta$-catenin-positive cases; there were no false positives by $\beta$-catenin IHC.

Emily M. Erstine

emilyerstine@gmail.com

1 Departments of Pathology, The University of Texas MD Anderson Cancer Center, 1515 Holcombe Blvd., Unit 85, Houston, TX 77030, USA

2 Departments of Genomic Medicine, The University of Texas MD Anderson Cancer Center, Houston, TX, USA

3 Departments of Dermatology, The University of Texas MD Anderson Cancer Center, Houston, TX, USA

4 Departments of Translational Molecular Pathology, The University of Texas MD Anderson Cancer Center, Houston, TX, USA
Deep penetrating nevus is a relatively recently defined diagnostic entity, formally defined as a diagnostic entity in 1989 by Seab and colleagues [2]. The typically darkly-pigmented lesions are most commonly located on the face, upper trunk, or proximal extremities of young female patients. Histologically, they demonstrate pleomorphic pigmented cells arranged in loose nests that penetrate deeply into the reticular dermis or subcutis. Nearly a third of the 70 cases studied by Seab et al. were initially misdiagnosed as melanoma, but there were no local recurrences or distant metastases.

Categorically speaking, DPN were regarded clinically and pathologically as a variant of blue nevus [3-5] until 2013 when Bender and colleagues showed that DPN harbor $H R A S$ mutations and lack recurrent activating mutations in GNAQ or GNA11 that blue nevi often possess [6]. While DPN may clinically appear like blue nevi, their pathologic distinction from benign blue nevi matters to the extent that it has been recently reclassified in the most recent $\mathrm{WHO}$ of skin tumors [7] as an intermediate melanocytic lesion alongside PEM. DPN possess some locoregional metastatic potential [8] distinguishing it from conventionally benign lesions, but not giving rise to distant metastases or death as seen in melanoma. The rare instances of lesions diagnosed as DPN associated with distant metastasis or death could well be melanoma misdiagnosed as DPN. Nevertheless, morphologic and molecular transformation from DPN to melanoma has been described on rare occasion [9]. Thus, it is very important to distinguish DPN from wholly benign melanocytic neoplasms and melanoma as their clinical management and prognosis are different. This present work suggests that immunohistochemistry for $\beta$-catenin could be a helpful tool within this differential diagnosis.

$\beta$-catenin is a key molecule in the complex Wnt signaling pathway, which regulates cell proliferation [10]. It appears to function primarily through the nucleus in mesenchymal and neuroectodermal tissues, but also has a membranous role with adherens junctions in epithelial tissues [11]. Dysregulation of the Wnt pathway via accumulation of $\beta$-catenin can occur by 
Fig. 1 Diagnostic workflow example for employing $\beta$-catenin immunohistochemistry as a screening tool for diagnostically ambiguous melanocytic neoplasms before extensive molecular analysis is performed. This workflow assumes that meticulous morphologic examination is performed before arriving at the conclusion that the lesion is a histologically ambiguous melanocytic neoplasm. Furthermore, it does not include a decision point for sending a case for expert consultation, which can occur at any point. Also, in the step of performing molecular analysis of lesions with nuclear accumulation of $\beta$-catenin by immunohistochemistry, cyclin D1 alterations are excluded. Abbreviations: IHC, immunohistochemistry; DPN, deep penetrating nevus; MAPK, MAP kinase

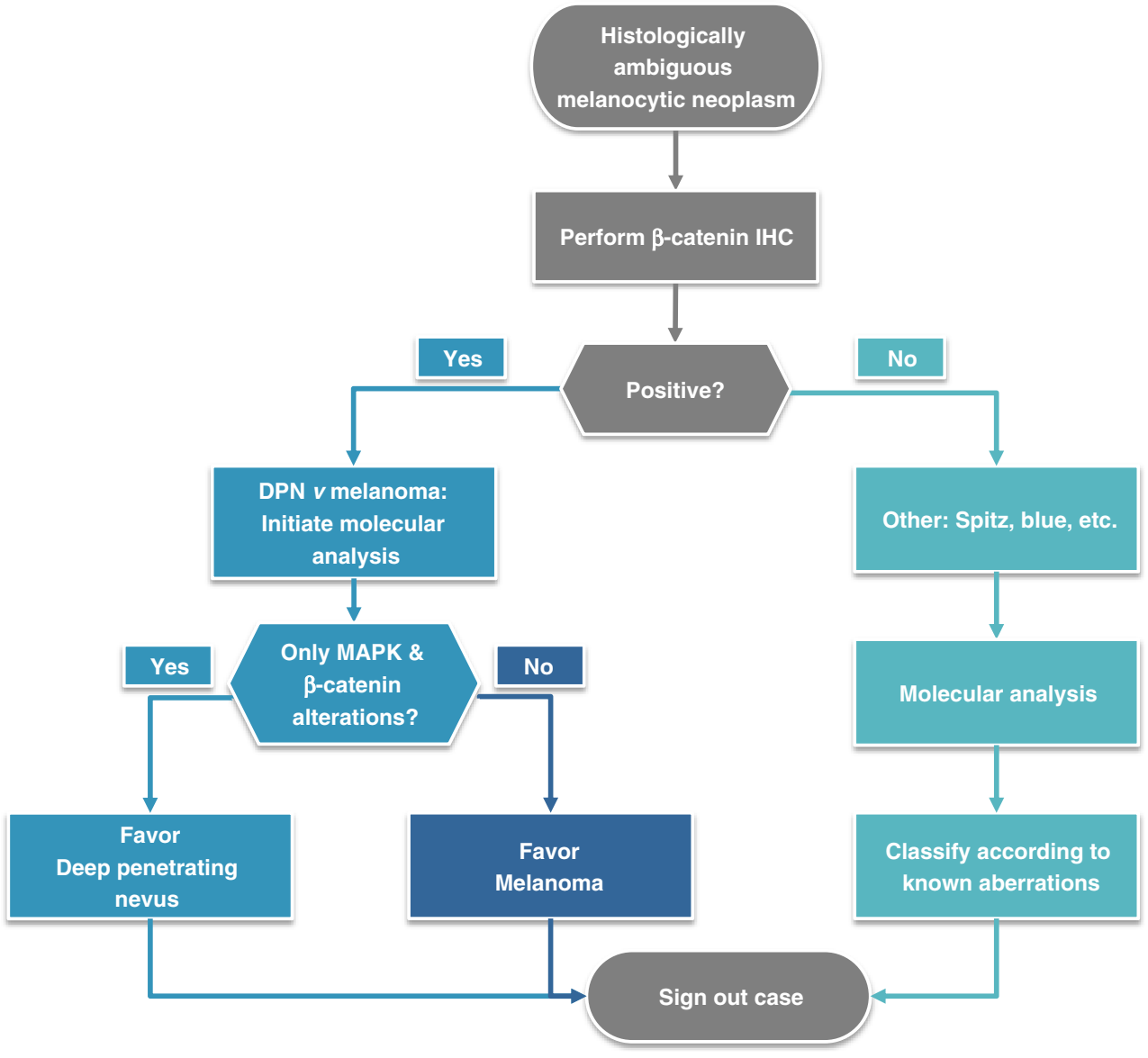

one of a variety of mechanisms, which in turn causes significant downstream effects, such as increased expression of cyclin D1, which is associated with various - usually malignant-neoplastic processes, including colorectal [12], hepatocellular [13] and pancreatic [14] carcinomas, melanoma, and dysplastic melanocytic nevi [15], to name a few.

Results of Fouchardiere et al.'s current work confirms their earlier work that alterations in the $\mathrm{Wnt} / \beta$-catenin pathway is one of the genetic anomalies that define DPN [16] and further suggests that $\beta$-catenin is a good screening tool prior to initiation of molecular analysis. Further validation of nuclear $\beta$ catenin IHC expression in a larger cohort of diagnostically ambiguous melanocytic lesions would be helpful to further elucidate any other diagnostic pitfalls of this very costeffective potential screening tool. Overcoming false positives, like those that occurred in the SSM and nevoid melanoma of the current study, should fall on careful histologic evaluation of melanocytic lesions in the majority of instances, while more extensive molecular studies such as multi-probe fluorescence in situ hybridization (FISH) or comparative genomic hybridization $(\mathrm{CGH})$ may need to be employed to resolve a minority of ambiguous cases. Furthermore, more extensive molecular analysis of the false-positive lesions may be warranted to assess a potentially more aggressive phenotype among SSM, nevoid melanomas, and others. Figure 1 proposes a practical diagnostic workflow for the melanocytic lesions discussed herein.

This work highlights how research on pathogenetic mechanisms can be rapidly translated for evaluation as a diagnostic aid in patient care. Certainly more work with increased case numbers and diversity is needed as it is the unfortunate natural history of virtually all biomarkers to decline in reported sensitivity and specificity as additional publications accumulate. As with all biomarkers, $\beta$-catenin should be assessed within the clinical and histologic context of its use and interpreted as part of a panel of biomarkers to avoid overinterpretation.

Acknowledgments We would like to thank Kim-Anh T. Vu for creating the graphic for this article.

Authors' contributions Alexander J. Lazar, $\mathrm{MD} / \mathrm{PhD}$, conceived the structure and guided the content of as well as edited the manuscript. Emily M. Erstine, MD, reviewed the literature and wrote and edited the manuscript. Both authors gave final approval for publication and take full responsibility for the work as a whole, including the decision to submit and publish the manuscript.

\section{Compliance with ethical standards}

The authors have fulfilled all ethical standards relevant for this manuscript. 
Conflict of interest The authors declare that they have no conflict of interest.

\section{References}

1. de la Fouchardière A, Caillot C, Jacquemus J, Durieux E, Houlier A, Haddad V, Pissaloux D (2019) $\beta$-Catenin nuclear expression discriminates deep penetrating nevi from other cutaneous melanocytic tumors. Virchows Arch. https://doi.org/10.1007/ s00428-019-02533-9

2. Seab J, Graham J, Helwig E (1989) Deep penetrating nevus. Am J Surg Pathol 13(1):39-44

3. Scolyer RA, Zhuang L, Palmer AA, Thompson JF, McCarthy SW (2004) Combined naevus: a benign lesion frequently misdiagnosed both clinically and pathologically as melanoma. Pathology 36(5): 419-427. https://doi.org/10.1080/00313020412331283879

4. Maize JC, McCalmont TH, Carlson JA, Busam KJ, Kutzner H, Bastian BC (2005) Genomic analysis of blue nevi and related dermal melanocytic proliferations. Am J Surg Pathol 29(9):12141220. https://doi.org/10.1097/01.pas.0000165527.01816.d1

5. LeBoit P, Burg G, Weedon D, Sarasin A, eds (2006) World Health Organization classification of tumours. Pathology and genetics of skin tumours. 1st edn. IARC, Lyon, pp 98-99

6. Bender RP, McGinniss MJ, Esmay P, Velazquez EF, Reimann JD (2013) Identification of HRAS mutations and absence of GNAQ or GNA11 mutations in deep penetrating nevi. Mod Pathol 26(10): 1320-1328. https://doi.org/10.1038/modpathol.2013.77

7. Elder D, Massi D, Willemze R, Al E, eds (2018) WHO classification of skin tumours. 4th edn. IARC, Lyon, pp 95-96

8. McCalmont TH, Bastian BC (2012) An unconventional deep penetrating melanocytic nevus with microscopic involvement of regional lymph nodes. J Cutan Pathol 39(1):25-28. https://doi.org/ 10.1111/j.1600-0560.2011.01850.x
9. Magro C, Abraham R, Guo R, Al E (2014) Deep penetrating nevuslike borderline tumors: a unique subset of ambiguous melanocytic tumors with malignant potential and normal cytogenetics. Eur J Dermatol 24(5):594-602

10. Nusse R, Clevers H (2017) Wnt/ $\beta$-catenin signaling, disease, and emerging therapeutic modalities. Cell 169(6):985-999. https://doi. org/10.1016/j.cell.2017.05.016

11. Valenta T, Hausmann G, Basler K (2012) The many faces and functions of Î 2-catenin. EMBO J 31(12):2714-2736. https://doi. org/10.1038/emboj.2012.150

12. Tetsu O, Mccormick F (1999) Beta-catenin regulates expression of cyclin D1 in colon carcinoma cells. Nature 398(6726):422-426

13. Gotoh J, Obata M, Yoshie M, Kasai S, Ogawa K (2003) Cyclin D1 over-expression correlates with $\beta$-catenin activation, but not with H-ras mutations, and phosphorylation of Akt, GSK $3 \beta$ and ERK1/2 in mouse hepatic carcinogenesis. Carcinogenesis 24(3):435-442

14. Georgiadou D, Sergentanis T, Sakellariou S, Al E (2014) Cyclin D1, p16(INK) (4A) and p27(Kip1) in pancreatic adenocarcinoma: assessing prognostic implications through quantitative image analysis. APMIS 122(12):1230-1239

15. Alekseenko A, Wojas-Pelc A, Lis GJ, Furgał-Borzych A, Surówka G, Litwin JA (2010) Cyclin D1 and D3 expression in melanocytic skin lesions. Arch Dermatol Res 302(7):545-550. https://doi.org/ 10.1007/s00403-010-1054-3

16. Yeh I, Lang UE, Durieux E, et al (2017) Combined activation of MAP kinase pathway and $\beta$-catenin signaling cause deep penetrating nevi. Nat Commun 8(1). https://doi.org/10.1038/s41467-01700758-3

Publisher's note Springer Nature remains neutral with regard to jurisdictional claims in published maps and institutional affiliations. 